\title{
Research Paper \\ The Effect of a Premarital Educational Program Based on 9- Type Personalities Types (Enneagram) on Emotional Expressiveness of Couples Preparing for Marriage
}

\author{
Farnaz Navabifar ${ }^{1} \odot{ }^{*}$ Hamid Atashpour $^{1} \odot$, Mohsen Golparvar $^{1} \odot$
}

1. Department of Psychology, Faculty of Psychology \& Educational Science, Isfahan (Khorasgan) Branch, Islamic Azad University, Isfahan, Iran.

\begin{tabular}{|c|c|}
\hline $\begin{array}{l}\text { Use vour device to scan } \\
\text { and read the article online }\end{array}$ & dtrat on Navabifar F, Atashpour H, Golparvar M. [The Effect of a Premarital Educational Program Based on 9- Type Per- \\
\hline 回福神回 & $\begin{array}{l}\text { sonalities Types (Enneagram) on Emotional Expressiveness of Couples Preparing for Marriage (Persian)]. Iranian Journal of } \\
\text { Psychiatry and Clinical Psychology. 2020:26(1):2-15. http://dx.doi.org/10.32598/iipcp.26.1.3128.1 }\end{array}$ \\
\hline prtistis & d si" http://dx.doi.org/10.32598/ijpcp.26.1.3128.1 \\
\hline
\end{tabular}

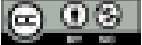

Received: 27 Jul 2019 Accepted: 12 Jan 2020 Available Online: 01 Apr 2020

Key words: Emotional expres siveness, Personality types, Enneagram, Premarital counseling

\begin{abstract}
A B S T R A C T
Objectives This study aimed to develop a premarital educational package based on based on 9-type personalities (Enneagram) to improve emotional expressiveness of couples preparing for marriage. Methods In this quasi-experimental study with pretest/posttest design, the study population consisted of all couples in the premarital stage referred to counseling centers in Isfahan, Iran during spring and summer 2017. Of these, 30 were selected and divided into two groups of intervention and control. They completed the Emotional Expressiveness Questionnaire (EEQ) before the study and 45 days after the end of intervention. The educational package was developed based on the study literature and techniques related to enneagram typology and considering its constructs, and then presented to the intervention at ten 90 -min sessions once a week.

Results The ANCOVA results indicated that the designed enneagram-based intervention could significantly affect the emotional expressiveness of the study couples. There was a significant difference between the scores of study groups after intervention $(P<0.05)$.

Conclusion the designed enneagram-based educational package was effective in improving the emotional expressiveness of couples preparing for marriage through familiarity with the personality traits of themselves and their partner. These results are useful for professionals and those involved in premarital counseling to provide better services.
\end{abstract}

\section{Extended Abstract}

\section{Introduction}

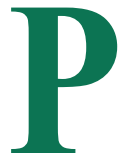

remarital preparation course is a new approach to prevent and eliminate dissatisfaction with marriage, where couples learn how to have a successful and lasting matrimony. Accordingly, premarital counseling is an educational, therapeutic, and preventive method. Preventive approaches have two major goals: improving family life and preventing divorce. The theories and models proposed in the field of marriage clarifies that various factors affect the stability of marriage. One of the most important factors is the personality traits of couples. On the other hand, maintaining romantic and satisfying relationships requires the ability to recognize and express emotions. Emotional expressiveness refers to a facial movement, bodily behavior, or verbal expression that communicates an emotional state in a special and reliable way.

Previous studies suggest that emotional expression is associated with the individuals' personality traits. One of the new topics in the field of typology in psychology, especially

* Corresponding Author: Hamid Atashpour, PhD.

Address: Department of Psychology, Faculty of Psychology \& Educational Science, Isfahan (Khorasgan) Branch, Islamic Azad University, Isfahan, Iran Tel: +98 (913) 1068707

E-mail: hamidatashpour@gmail.com 


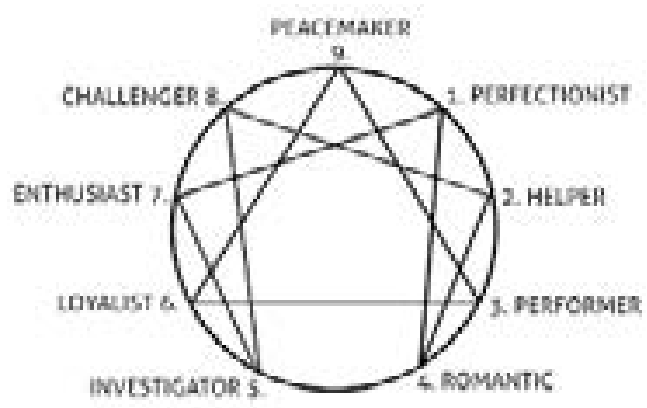

Figure 1. The enneagram of personality types

in Iran is the nine-personality types known as "enneagram". It is a system consisting of nine personality types that addresses the strengths and weakness of each type and provides a global language that helps people better understand themselves and others. The enneagram diagram consists of a triangle and a hexagon enclosed within a circle. The combination of these elements creates nine points along the circle. Each of these nine points provides a strategy for interacting with the environment which affects personality and identifies nine distinct personality types (Figure 1).

The study of emotional expression in couples applying for marriage is important, theoretically and functionally, because our greater understanding of the personality traits that influences emotional expression can lead to a better understanding of this phenomenon, especially in the premarital phase. To the best of our knowledge, no enneagram-based interventional program has been developed to affect the emotional expression of couples. Thus, the present study is the first study aimed to develop an educational package based on enneagram types to affect the emotional expressiveness of couples preparing for marriage.

\section{Methods}

\section{Study subjects}

This quasi-experimental study was conducted on 30 couples in premarital phase selected using a convenience sampling technique and randomly assigned into intervention and control groups, after meeting the inclusion and exclusion criteria. The inclusion criteria were: having a high school degree, not attending other premarital educational courses before entering the study, age 20-35 years, and no previous marriage and training. The exclusion criteria including: absent for more than two training session, being under both training and counseling, and having any mental disease that can prevent attending training sessions according to Diagnostic and Statistical Manual of Mental Disorders, $5^{\text {th }}$ Edition (DSM-5) criteria diagnosed by a psychia-
Iranian Journal of

PSYCHIATRY AND CUINICAL PSYCHOLOGY

trist. The subjects in both groups completed the Emotional Expressiveness Questionnaire (EEQ) before the study, and 45 days after the end of intervention. The educational package was presented to the intervention group at ten 90-min sessions once a week. The control group received no information but was informed to receive the package later.

\section{Developing the educational Package}

The enneagram-based educational package was developed based on the study literature, the emotional expression predictors, and existing enneagram principles provided by experts in Iran and other countries. Then, the objectives, contents, and assignments were evaluated. By combining and matching techniques and the related emotional expression components including behaviors, attitudes and views, needs and desires, fears and concerns, and defense mechanisms, a guideline was formulated entitled "A guide to the enneagram with a focus on emotional expression", aiming to affect the emotional expressiveness of marriage applicants. Then, it's the initial packages were distributed among five premarital consultants with a $\mathrm{PhD}$ degree in premarital education, and were asked to rate the structure and content of each session based on a Likert scale. The final version was then confirmed after modifications. Then, the effect of the designed package on the emotional expressiveness of couples was assessed using ANCOVA.

\section{Results}

The results indicated that the designed enneagram-based intervention could significantly affect the emotional expressiveness of the study couples. There was a significant difference between the scores of intervention and control groups after intervention $(\mathrm{P}<0.05)$ (Table 1).

\section{Discussion}

The enneagram-based educational package could significantly affect the behaviors, attitudes and views, needs and 
Table 1. ANCOVA results for the effect of intervention on emotional expressiveness

\begin{tabular}{|c|c|c|c|c|c|c|c|c|}
\hline Variable & Source & $\begin{array}{l}\text { Sum of } \\
\text { Square }\end{array}$ & df & Mean Square & $\mathbf{F}$ & Sig. & $\begin{array}{l}\text { Partial Eta } \\
\text { Squared }\end{array}$ & $\begin{array}{c}\text { Observed } \\
\text { Power }\end{array}$ \\
\hline \multirow{3}{*}{$\begin{array}{l}\text { Expression of positive } \\
\text { emotion }\end{array}$} & Pre-test & 198.84 & 1 & 198.84 & 0.22 & 0.64 & 0.004 & 0.07 \\
\hline & Group & 130914.55 & 1 & 130914.55 & 148.26 & 0.000 & 0.73 & 1 \\
\hline & Error & 47680.97 & 54 & 882.98 & - & - & - & - \\
\hline \multirow{3}{*}{$\begin{array}{l}\text { Expression of inti- } \\
\text { macy }\end{array}$} & Pre-test & 3490.51 & 1 & 3490.51 & 3.16 & 0.8 & 0.11 & 0.704 \\
\hline & Group & 78023.92 & 1 & 78023.92 & 70.67 & 0.000 & 0.56 & 1 \\
\hline & Error & 56613.9 & 54 & 1103.96 & - & - & - & - \\
\hline \multirow{3}{*}{$\begin{array}{l}\text { Expression of nega- } \\
\text { tive emotion }\end{array}$} & Pre-test & 24.42 & 1 & 24.42 & 0.02 & 0.89 & 0.000 & 0.05 \\
\hline & Group & 82716.39 & 1 & 82716.39 & 66.58 & 0.000 & 0.55 & 1 \\
\hline & Error & 67090.92 & 54 & 12421.42 & - & - & - & - \\
\hline \multirow{3}{*}{ Overall } & Pre-test & 1232.41 & 1 & 1232.41 & 1.77 & .019 & 0.032 & 0.26 \\
\hline & Group & 141655.27 & 1 & 141655.27 & 203.29 & 0.000 & 0.80 & 1 \\
\hline & Error & 37627.05 & 37627.05 & 696.8 & - & - & - & - \\
\hline
\end{tabular}

desires, fears and concerns, defense mechanisms and, consequently, the emotional expressiveness of couples. These findings support the results of previous studies. Since the enneagram-based tasks continued after the end of intervention and the couples were supposed to perform them at home, it was observed that after 45 days the improvements were significantly continued, indicating the stable effect of the educational intervention. It is suggested that further studies should be conducted tp compare the effect of enneagram-based educational package on the emotional expressiveness of couples with the effect of other personality-related educational tools.

\section{Ethical Considerations}

\section{Compliance with ethical guidelines}

This study obtained its ethical approval from the Research Ethics Committee of Islamic Azad University of Khorasgan branch (Code: IR.IAU.KHUISF.REC.1397.058). The participants were informed about the study objectives and methods and were also assured of the confidentiality of their information. They were allowed to leave the study at any time.

Funding

This study was extracted from the $\mathrm{PhD}$. thesis of the first author approved by the Department of Psychology, Faculty of Psychology \& Educational Science, Isfahan (Khorasgan) Branch, Islamic Azad University.

\section{Authors contributions}

Conceptualization, data analysis, project administration: All authors; Methodology: Mohsen Golparvar, Farnaz Navabifar; Investigation, resources, initial draft preparation, editing and review: Farnaz Navabifar.

\section{Conflicts of interest}

The authors declared no conflict of interest. 


\title{
ساخت وبررسى اثربخشى بسته آموزشى مبتنى بر تيبهاي شخصيتى نُكَّانه (انياتر ام) بر ابرازترى هيجان متقاضيان ازدواج
}

\author{
فرناز نوابىفر' • "خميد آتشيور' •، محسن كليرور'
}

1. كروه روانشناسى و علوم تربيتى، دانشكده علوم انسانى، دانشكاه آزاد اسلامى واحد اصفهان (خوراسكان)، اصفهان، ايران.

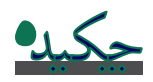

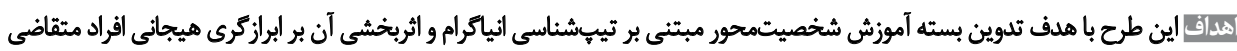

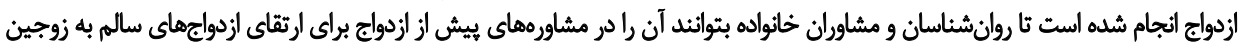
آموزش دهند.

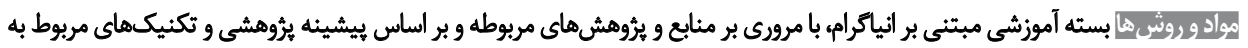

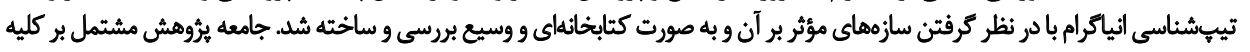

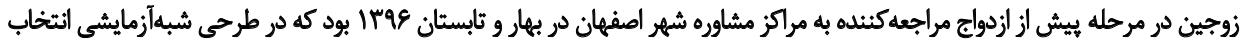

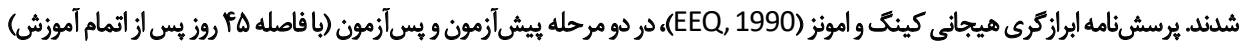

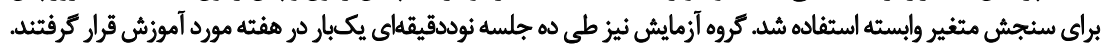

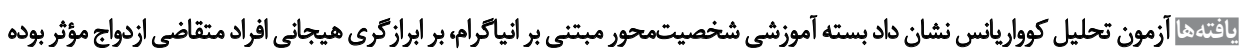

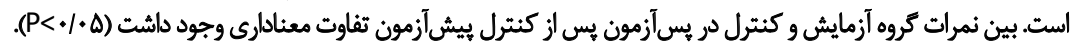

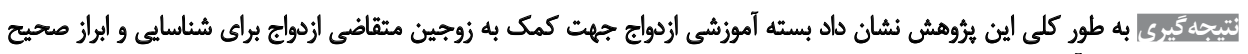

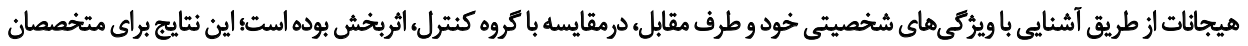

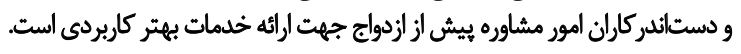

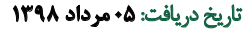

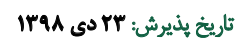

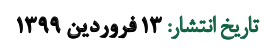

كليدوازوها:

ابرازكرى هيجاني، متقاضيان

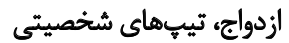

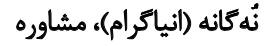

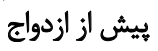

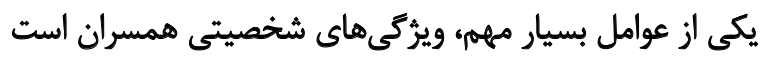

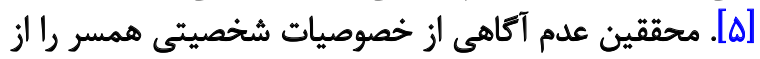

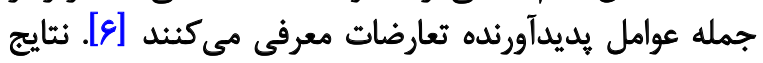

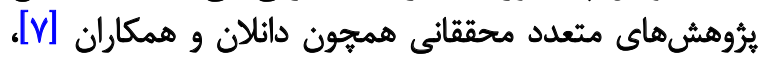

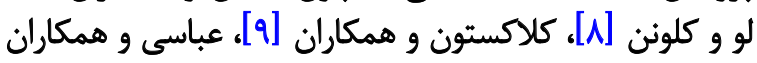

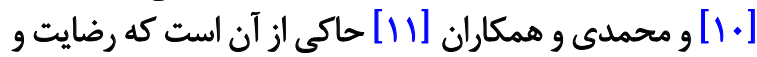

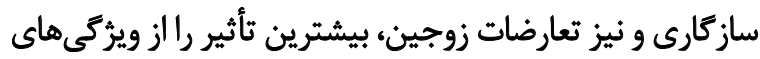

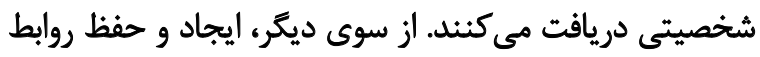

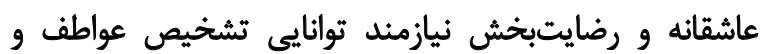

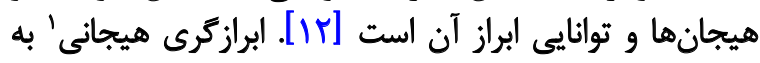

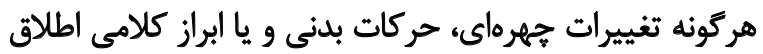

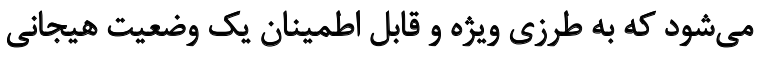

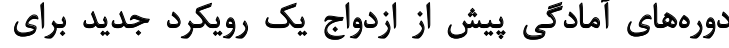

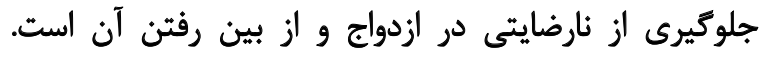

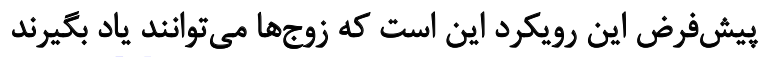

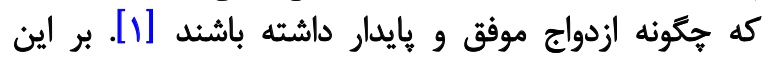

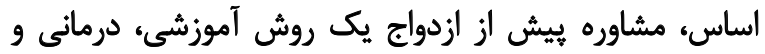

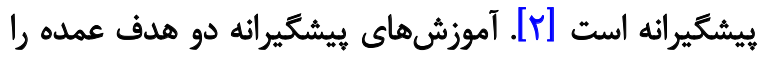

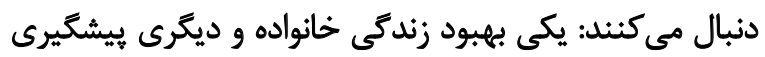

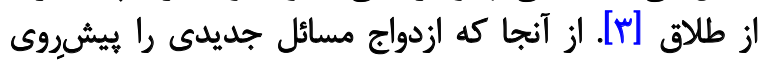

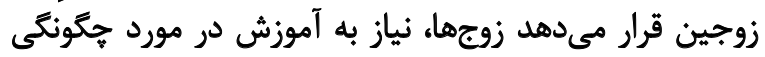

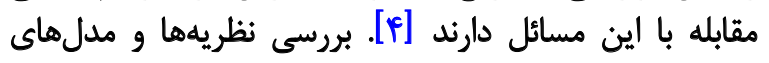

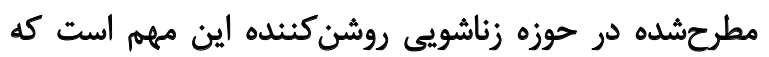

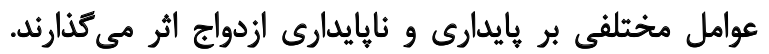

\section{드.}

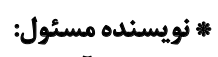

كتتر حميد آتشيور

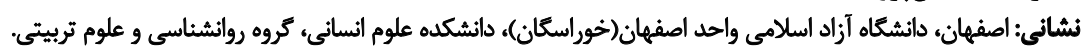
تلفئ: بيست الكترونيكى: hamidatashpour@gmail.com 
ويرُكى هاى شخصيتى تأثيركذار بر ابرازگرى هيجان مى بتواند

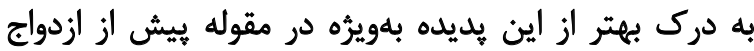

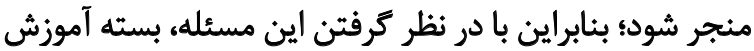

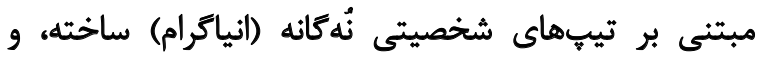

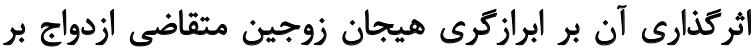

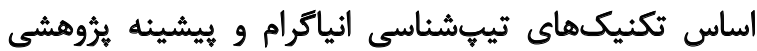

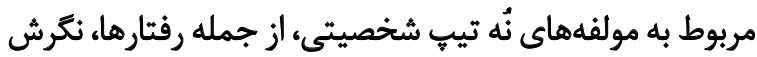

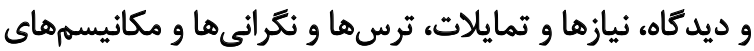

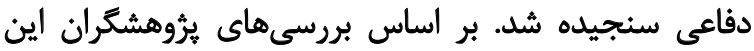

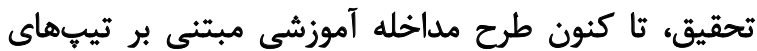

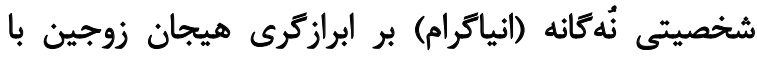

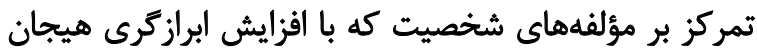

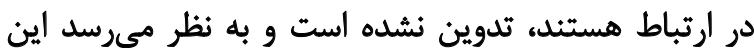
يروهش اولين طرح موجود در اين حوزه باشد.

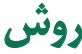

اين يُروهش از نظر دستهبندى بر مبناى هدف، از نوع

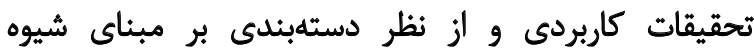

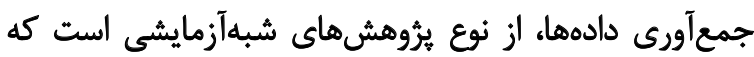

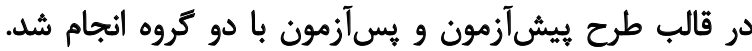

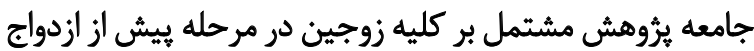

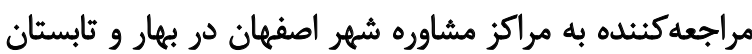

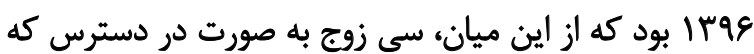

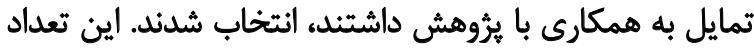

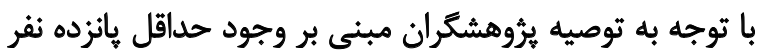

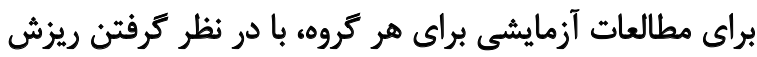

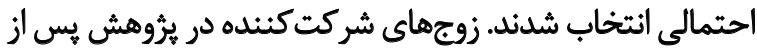

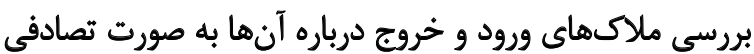
به دو كروه آزمايش و كنترل (هركدام هانزده زوج) تقسيم شدرند. ملاكهاى ورود شركتكنندهها عبارت بودند ازئ رضايت

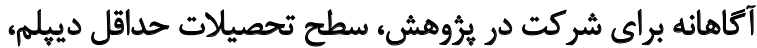

را منتقل مى كند [با]. ابرازگرى هيجانى با برخى از فرايندهاى

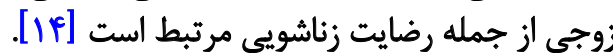

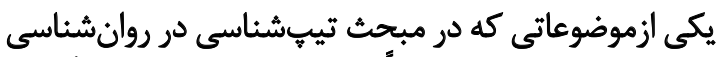

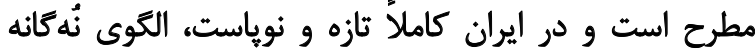

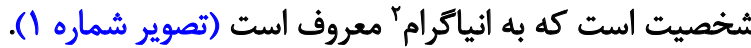

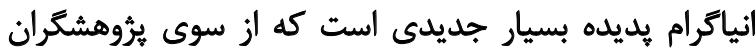

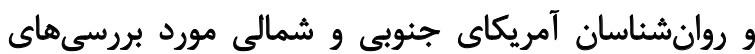

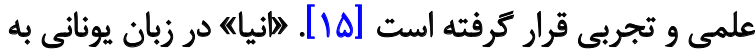

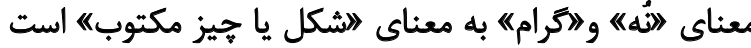

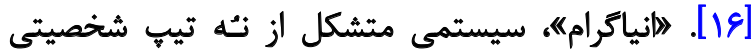

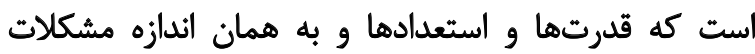

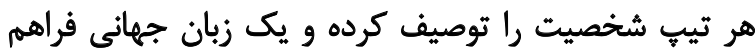

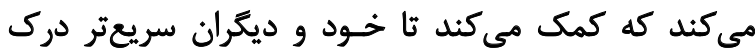

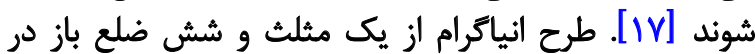

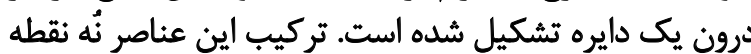

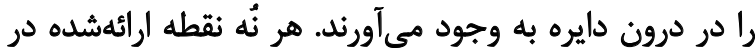

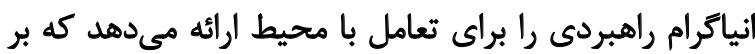

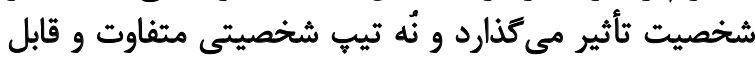

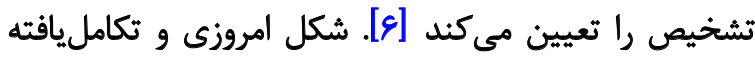

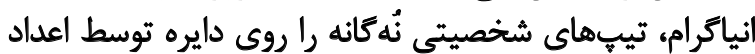

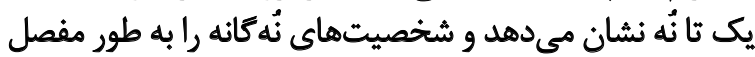

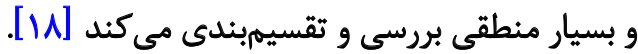
با در نظر كرفتن رويكرد بيشكيرانه آموزشهاى بيش ازيش ازئن

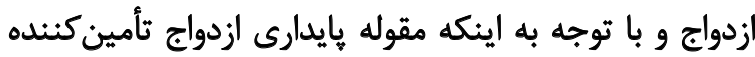

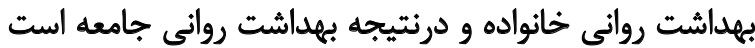

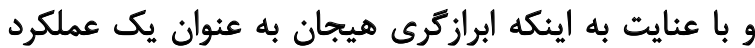

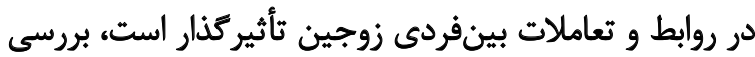

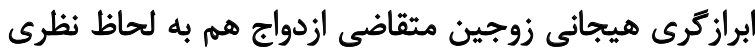

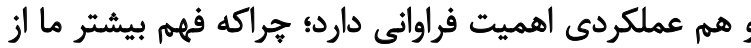

\section{Enneagram}

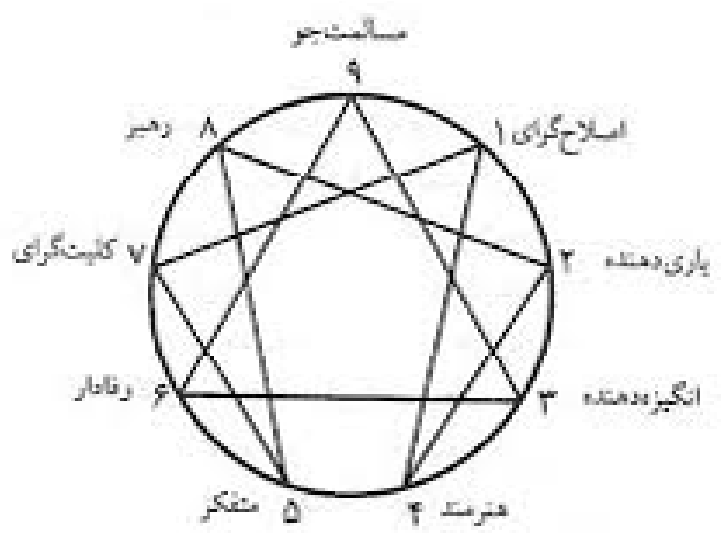




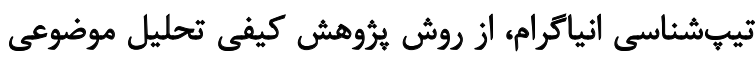

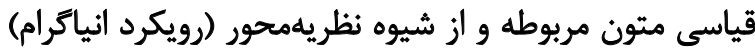

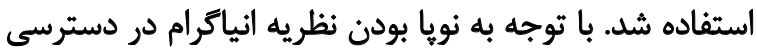

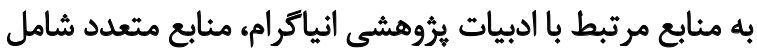

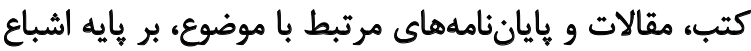

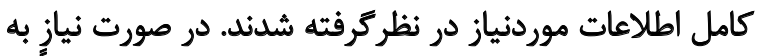

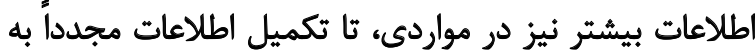

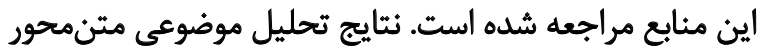

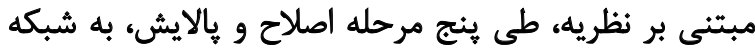

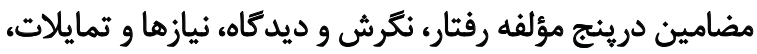

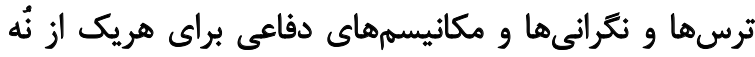

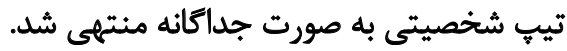

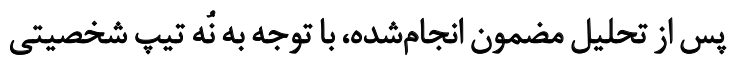

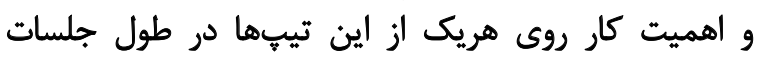

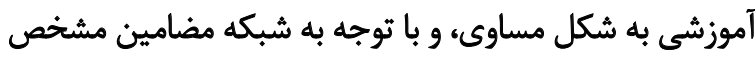

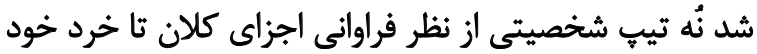

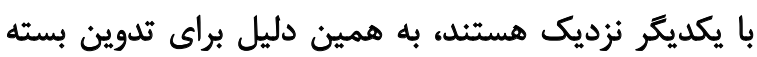

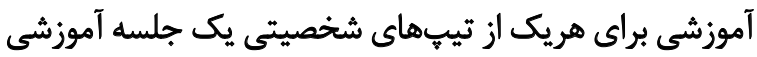

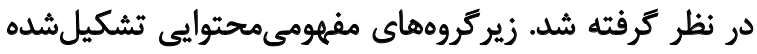

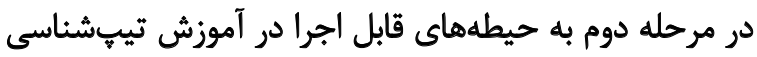

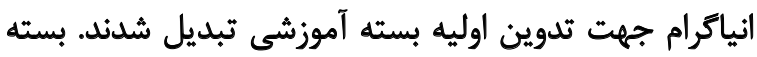

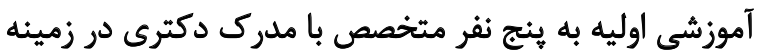

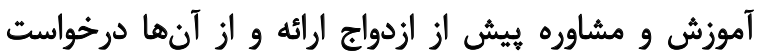

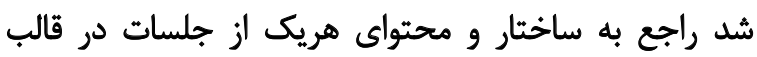

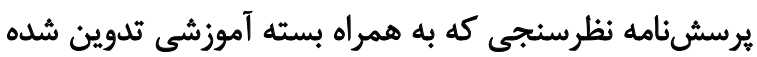

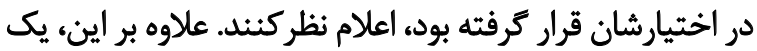

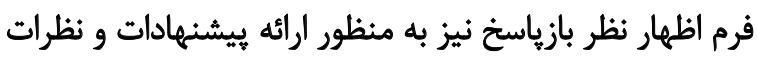

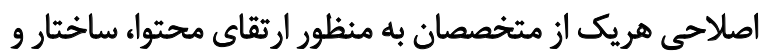

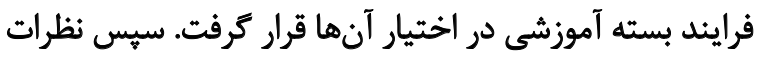

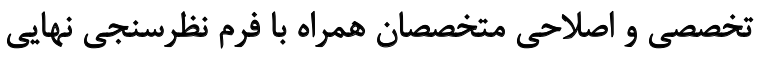

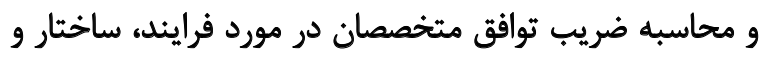

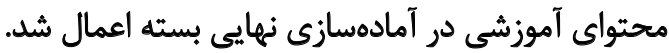
ضريب توافق بر سر محتواى جلسات براى آموزش انياكرام

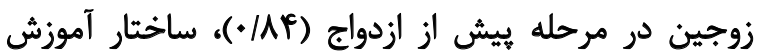

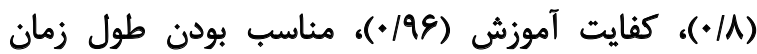

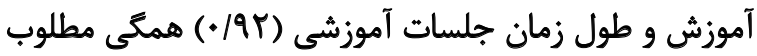

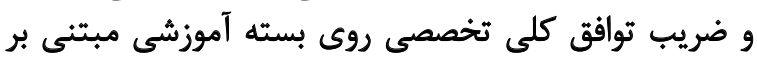

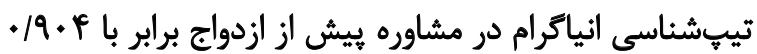

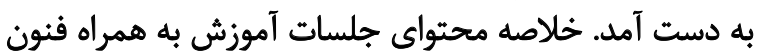

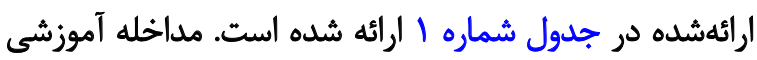

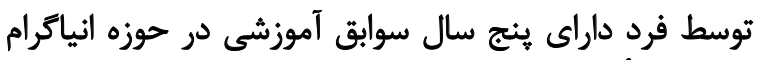

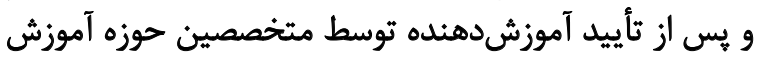

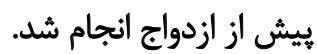

عدم حضور دركلاس هاى آموزش بيش از ازدواج قبل از ورود

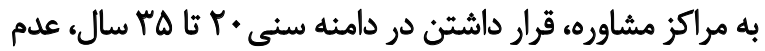

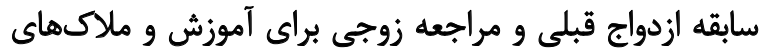

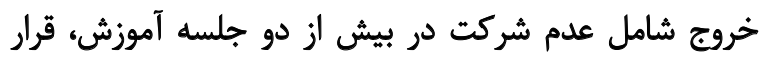

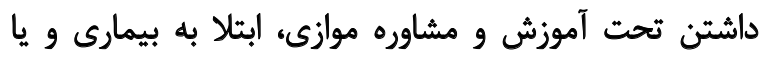

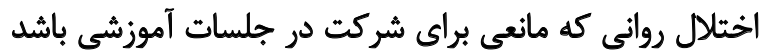

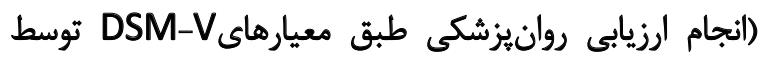

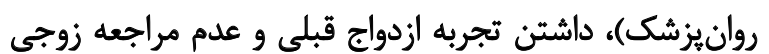

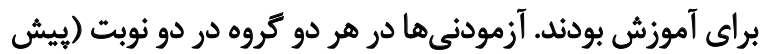

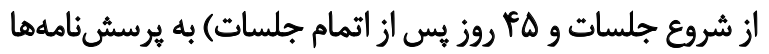

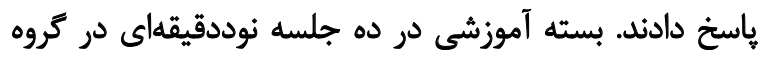

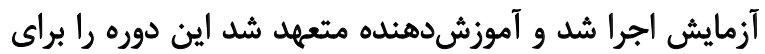
افراد كروه كنترل در جهند ماه آينده بركزار كند.

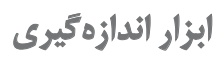

يرسشنامه ابراز ترى هيجانى كينك و امونز”

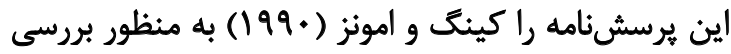

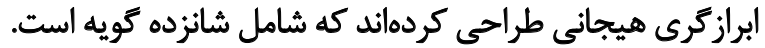

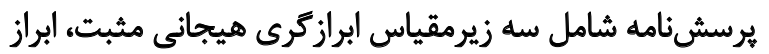

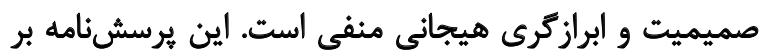

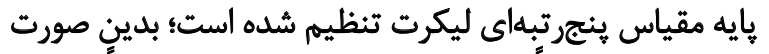

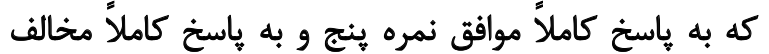

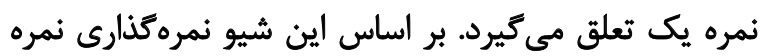

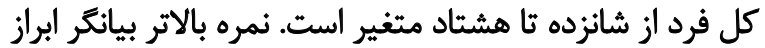

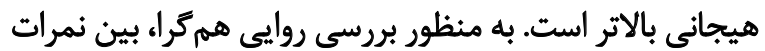

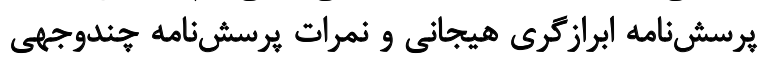

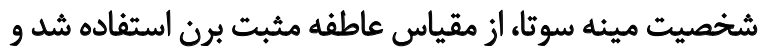

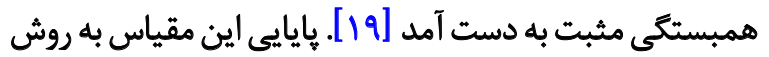

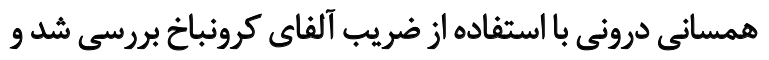

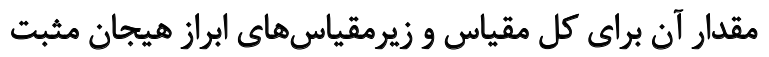

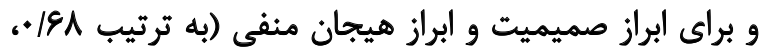

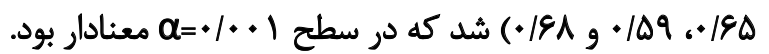

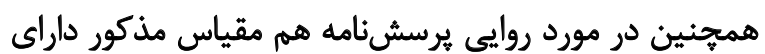

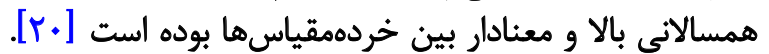

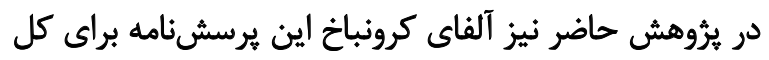

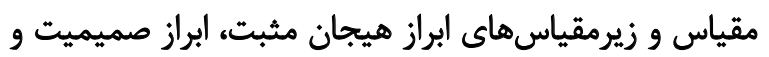

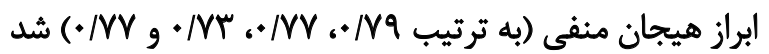

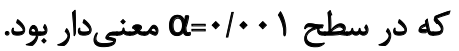
روش ساخت بسته آموزشى به منظور تدوين و آمادهازى بسته آموزشى مبتنى بر

3. Emotional Expressiveness Questionnaire (EEQ) 
جدول ا. شرح اختصارى جلسات آموزش مبتنى بر تيبشناسى انياترام در آموزش بيش از ازدواج

\begin{tabular}{|c|c|c|c|}
\hline همتواى جلسه و فنون و تكنيكها & هدف جلسه آموزش & عنوان كلى جلسه & 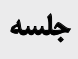 \\
\hline 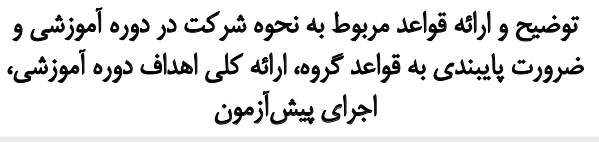 & آشنايى و معارفه مقدماتيى & 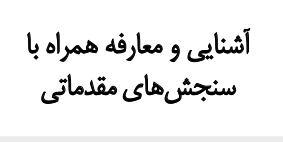 & 9 \\
\hline 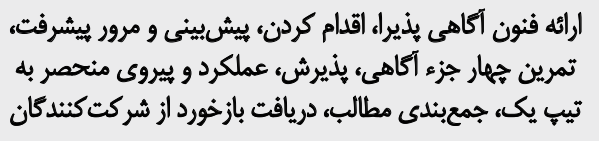 & 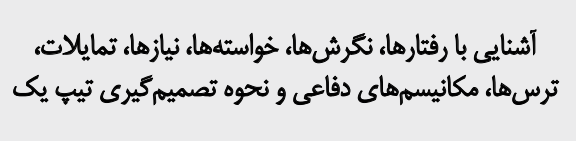 & أشنايى با تيبي شخصيتى & के \\
\hline 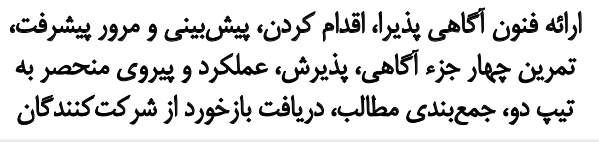 & 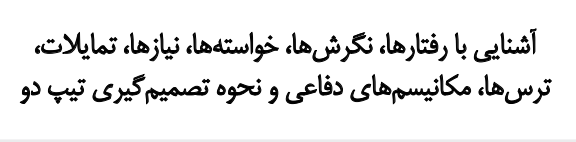 & أشنائي با تيب شخصيتى دو & 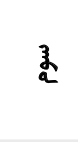 \\
\hline 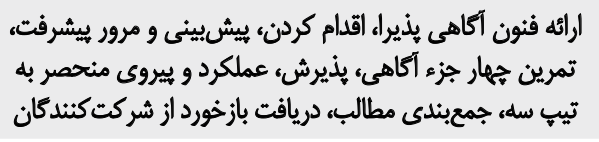 & 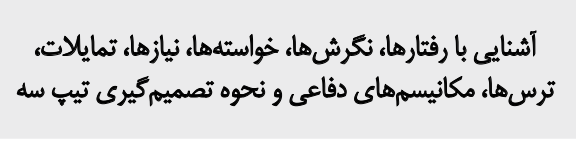 & أشنايع با تيب شخصيتى & 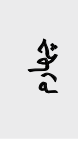 \\
\hline 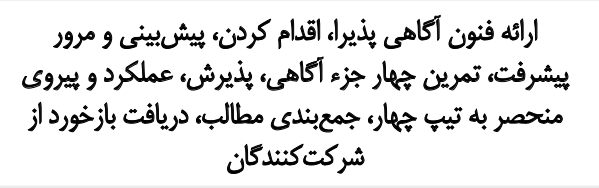 & 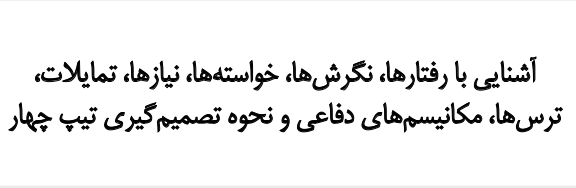 & أشنايى با تيبيَ شخصيتى & 象 \\
\hline 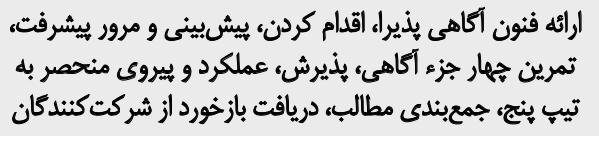 & 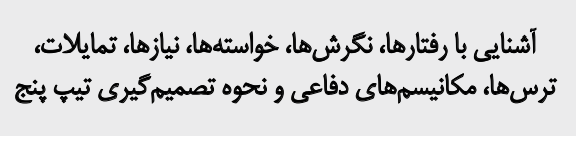 & أشنايي با تيبة شخصيتي & ? \\
\hline 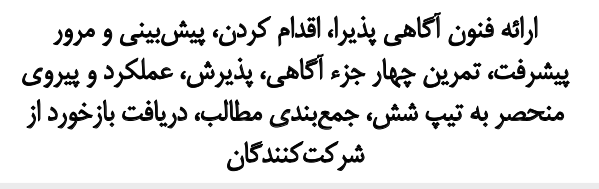 & 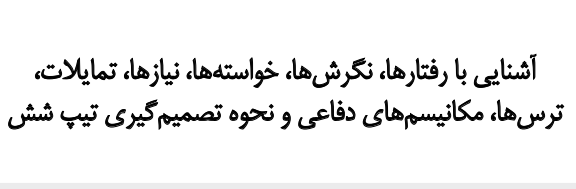 & أشنايع با تيبي شخصيتي & శ్రి \\
\hline 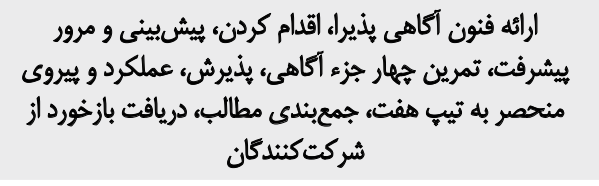 & 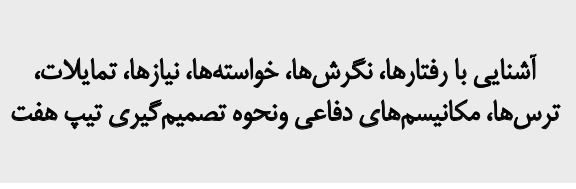 & آشنايي باتيب شخصيتي & 4 \\
\hline 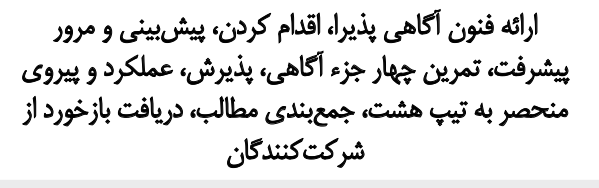 & 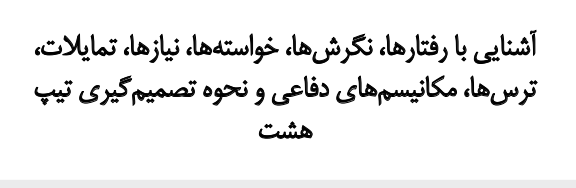 & أشناييى باتيب شخصيتى & $\frac{2}{2}$ \\
\hline 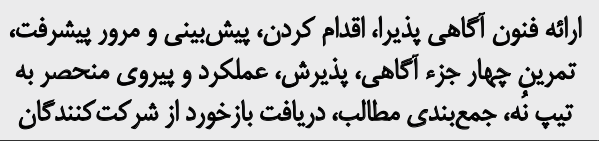 & 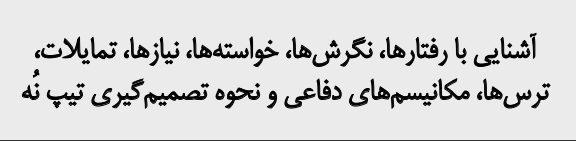 & أشنايع با تيب شخصيتى نُه & 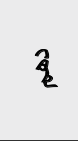 \\
\hline
\end{tabular}

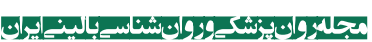

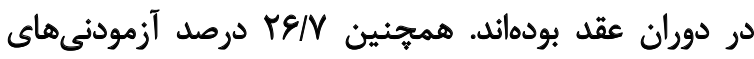

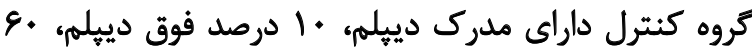

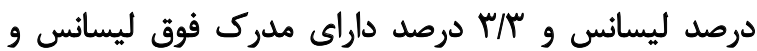

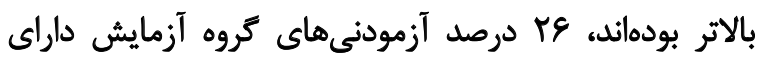

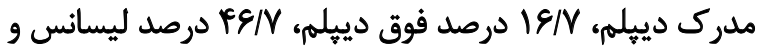

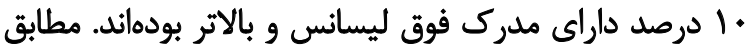

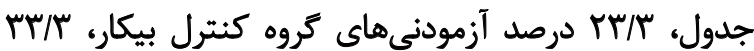

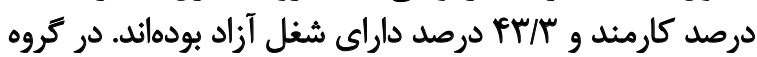

يافتهها جنان كه در جدول شماره Y مشاهده مي شودا،ينجاه درصد

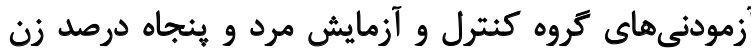

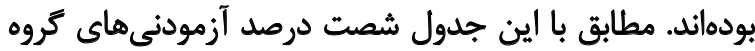

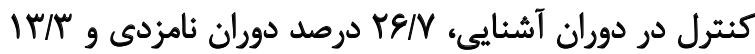

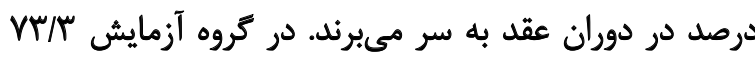

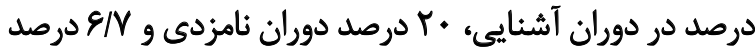


جدول r. توزيع فراوانى و درصد فراوانى كروهاى يرؤهى در متغيرهاى جمعيتشناختى

\begin{tabular}{|c|c|c|c|c|}
\hline \multirow{2}{*}{ خى دو (معنادارى) } & كروه آزمايش & كروه كتترل & \multirow{2}{*}{\multicolumn{2}{|c|}{ متغير }} \\
\hline & تعداد ( درصد فراوانى) & تعداد (درصد فراوانى) & & \\
\hline \multirow{2}{*}{ (1). } & $10(\Delta+)$ & $1 \theta(\Delta \cdot)$ & 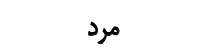 & \multirow{2}{*}{ جنسيت ل } \\
\hline & $1 \Delta(\Delta \cdot)$ & $10(\Delta \cdot)$ & زن ان & \\
\hline \multirow{3}{*}{$\left(\cdot / M^{9}\right) 1 / / r$} & $\pi(n / M)$ & $M(\xi)$. & دوره آشنايع & \multirow{3}{*}{ مرحله ازدواج } \\
\hline & $\varepsilon(r \cdot)$ & $\Lambda(r / N)$ & دوره نامزدى & \\
\hline & $r(\varepsilon / N)$ & $f(1 r / T)$ & دوره عقد & \\
\hline \multirow{3}{*}{$(. / 9.1)+(1)$} & $9(\mu \cdot)$ & $V(T / / Y)$ & بيكار & \multirow{3}{*}{ شعثل } \\
\hline & $r(M / / \mu)$ & $1 \cdot(\pi / r)$ & كارمند & \\
\hline & $I f(P S M)$ & $\pi(r / 4)$ & آزاد & \\
\hline \multirow{4}{*}{$(\cdot / 4 \varepsilon) r$} & $\Lambda(R \& M)$ & $\Lambda(K E M)$ & دييلم & \multirow{4}{*}{ تحصيلات } \\
\hline & $\Delta(1 \varepsilon / M)$ & $r(1 \cdot)$ & فوق دييلم & \\
\hline & If $(F E / M)$ & $M(\xi \cdot)$ & ليسانس & \\
\hline & $r(1)$. & $1(r / M)$ & فوق ليسانس و بالاتر & \\
\hline
\end{tabular}

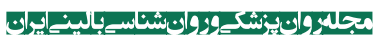

هيجان زوجين متقاضى ازدواج بررسى شد. ميانكين و اتحراف

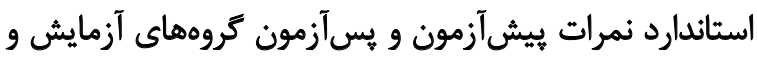

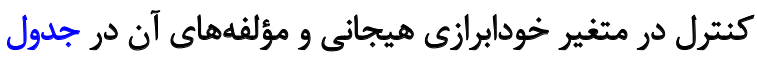
شماره ب به تفكيك ارائه شده است.

نتايج جدول شماره ب نشان مىدهد كه در متغير ابرازكرى سمان

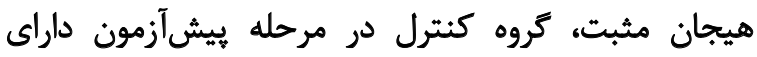

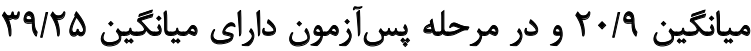

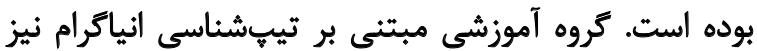

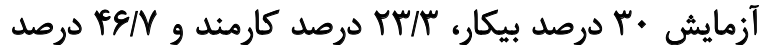

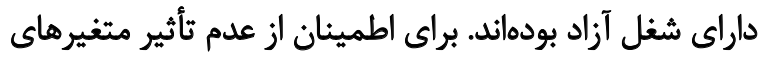

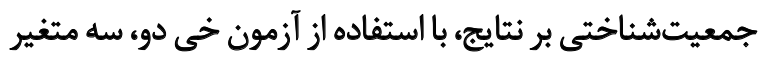

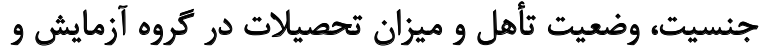

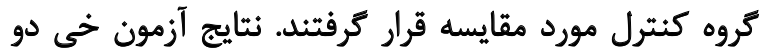

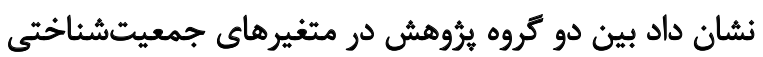

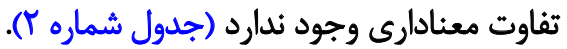
در اين بخش اثربخشى بسته تدوينشده بر ابرازگرى

جدول r. ميانكين و انحراف استاندارد خودابرازى هيجانى و مؤلفههاى آن در دو كروه يُروهش

\begin{tabular}{|c|c|c|c|}
\hline كروه آزمايش & كروه كتترل & محله & متغ \\
\hline ميانكين \ انحراف معيار & ميانكين \ انحراف معيار & & منير \\
\hline $\begin{array}{c}r \llbracket \pm \Delta / q r \\
\mid r q / . Y \pm r q / \star \Delta\end{array}$ & $\begin{array}{l}r \cdot / q \pm \Delta / F V \\
r q / T \Delta \pm r v / q T\end{array}$ & يبي آزآمون & ابرازٔرى هيجان هثبت \\
\hline 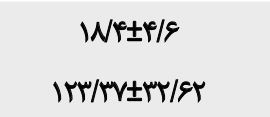 & 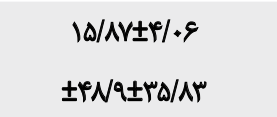 & يس آز آزمون & ابراز صميميت \\
\hline $\begin{array}{l}1 . / M^{\top} \pm T / g 9 \\
I r \Delta / N \pm r \Delta / N r\end{array}$ & $\begin{array}{c}1+/ \& \pm T / \Delta \\
\Gamma \& / \& \Delta \pm T r / \& \Delta\end{array}$ & يبي آزآمون & ابرازگرىى هيجان منفى \\
\hline 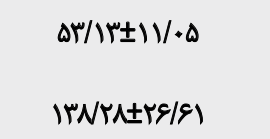 & $\begin{array}{l}r V / T Y \pm V / \Delta \Delta \\
r g / T \pm T V / N T\end{array}$ & يس آز آزمون & ابرازكرى هيجانى كل \\
\hline
\end{tabular}

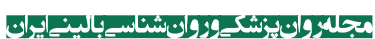


جدول F. نتايج آزمون تحليل كوواريانس مربوط به تأثيرمداخله آموزشى بر نمرات يس آزمون ابرازگرى هيجانى

\begin{tabular}{|c|c|c|c|c|c|c|c|c|}
\hline توان آمارى & ضريب اتا & سطح معنا دارى & مقدار F & ميانكين مجذورات & درجه آزادى & مجموع مجذورات & منيع تغييرات & متغير \\
\hline $.1 . V$ & $1 . .+F$ & . Iar & $\cdot / \pi r$ & I WAF & 1 & IQWAF & ييش آزمون & \\
\hline 1 & $\cdot / n$ & $\%$ & IFNTE & $|r+9| \varphi / \Delta \Delta$ & 1 & $\mid r \cdot q 1 \% / \Delta \Delta$ & كروهى عضى & هيجان مثبت \\
\hline- & - & - & - & $M T / u$ & $\Delta f$ & PVEA./QY & خطا & \\
\hline$\cdot N \cdot Y$ &.$/ 11$ &.$/ \Lambda$ & $r / / \varphi$ & $m p q . / \Delta 1$ & 1 & $m p q . / \Delta 1$ & ييش آزمون & \\
\hline 1 & $+/ \Delta \varepsilon$ & $* \ldots$ & $V . / 9 V$ & YA+Kr/qT & 1 & VA+Kr/qT & كروهى & ابراز صميميت \\
\hline- & - & - & - & $11 . r / q$ & $\Delta f$ & $\Delta 881 \% / 9$ & خطا & \\
\hline .1 .0 & $\%$ & .119 &.$/ r$ & $r e / 4 r$ & 1 & $r e / 4 r$ & هيش أزمون & \\
\hline 1 &.$/ \Delta \Delta$ & $\%$ & $98 / \Delta 1$ & ArrIs/rqq & 1 & ArrIF/Ta & كروهيت & هيجان منفى \\
\hline- & - & - & - & IMPYI/PY & $\Delta f$ & $9 V .9 . / 9 Y$ & خطا & \\
\hline e & $.1 . \pi$ & .119 & $1 / w$ & $|M T / P|$ & 1 & $|m T / P|$ & ييش أزمون & \\
\hline 1 & $+/ 1$ & $\%$ & $r \cdot r / r q$ & $|F| \& Q \Delta / T Y$ & 1 & $|F| \& \Delta \Delta / T Y$ & كروهيى & هيجانى كل \\
\hline - & - & - & - & $s q s / 1$ & VVGTVI+D & TVGTV/.D & خطا & \\
\hline
\end{tabular}

هيجانى كل، بين كروه آموزشى مبتنى بر تيبشناسى انياكرام

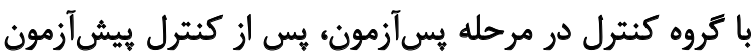

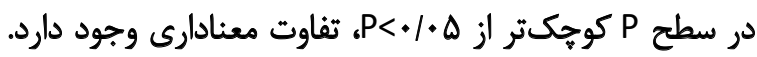

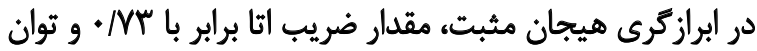

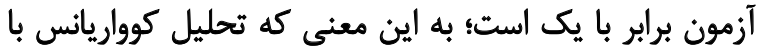

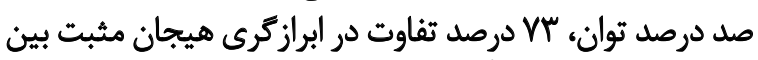

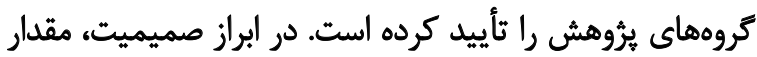

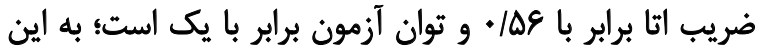

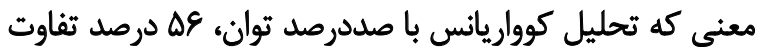

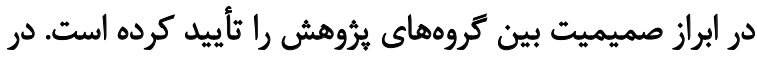

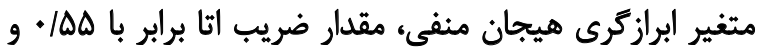

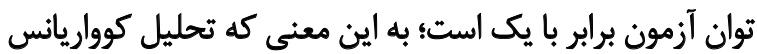

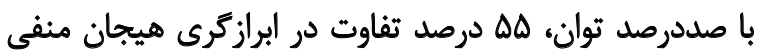

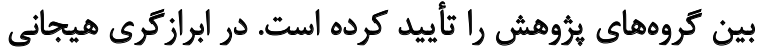

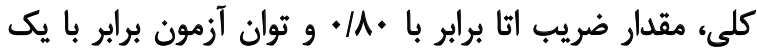

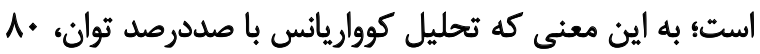

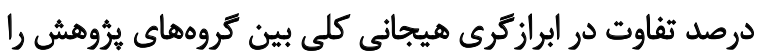

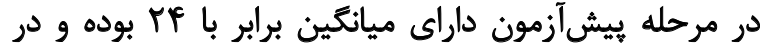

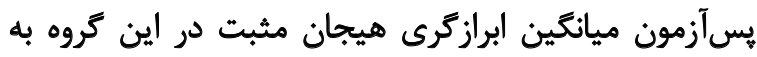

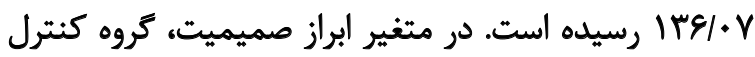

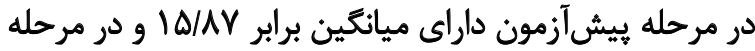

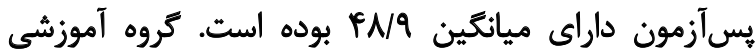

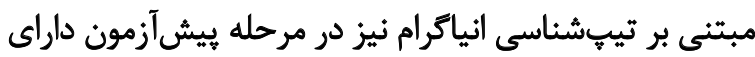

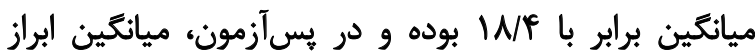

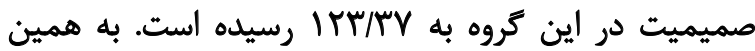

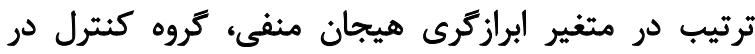

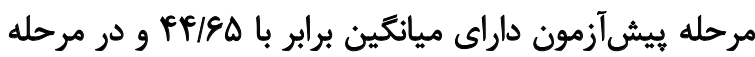

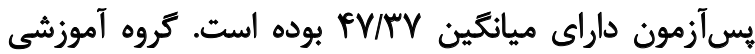

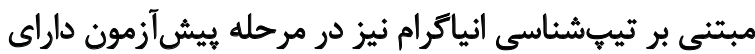

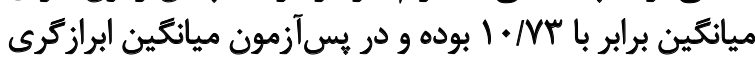

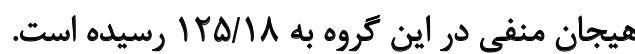

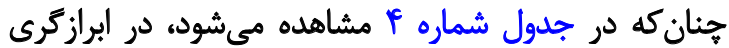

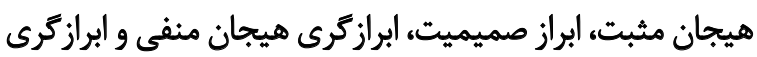




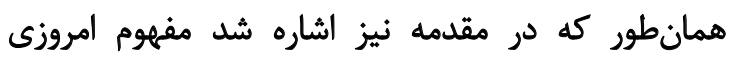

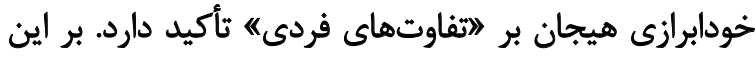

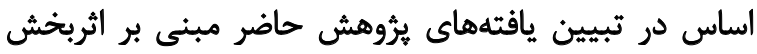

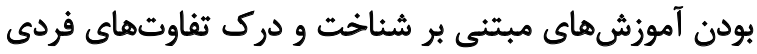

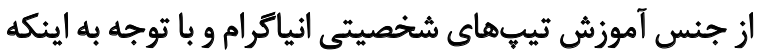

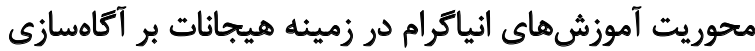

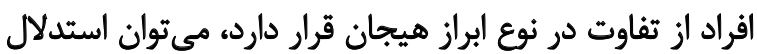

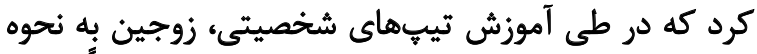

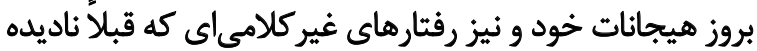

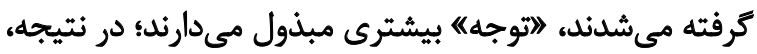

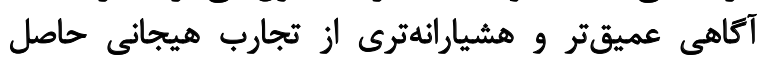

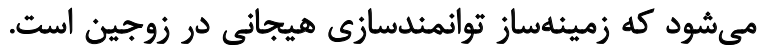

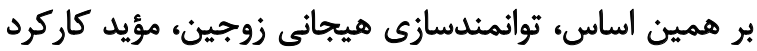

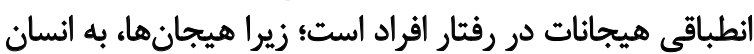

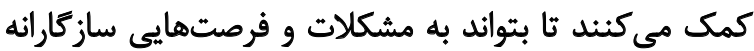

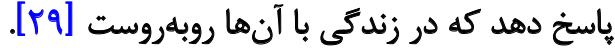
علاوه بر اين، نظريه انياكرام اصولى دارد كه با توصيف

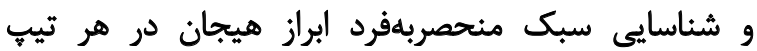

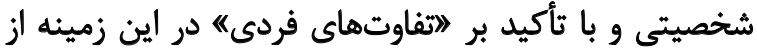

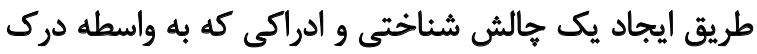

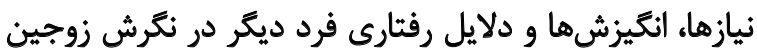

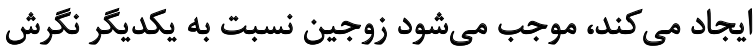

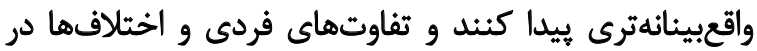

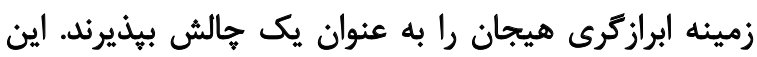

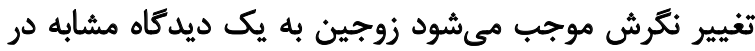

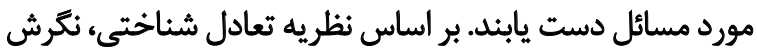

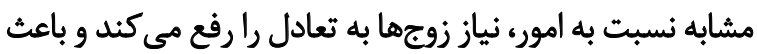

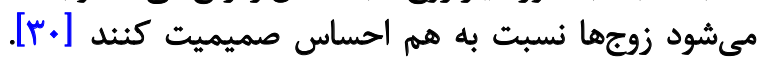

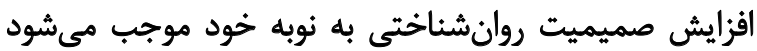

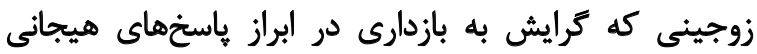

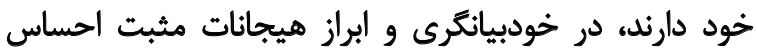

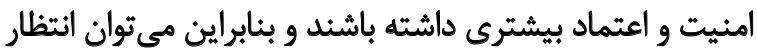

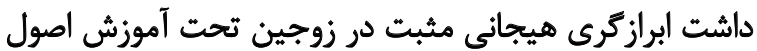
تيڤشناسى انياكرام به طور معنادارى مؤثر واقع شود. در تبيين يافته ديكر يروهش مبنى بر افزايش صميميث از إز إنيا

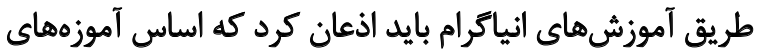

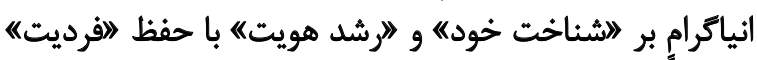

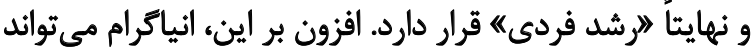

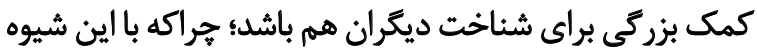

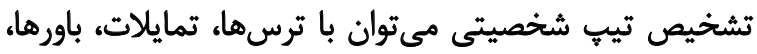

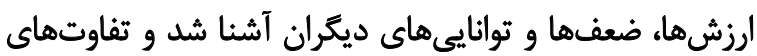

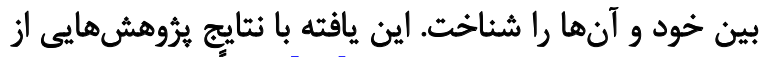

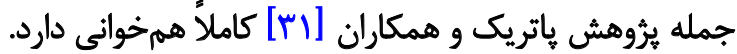

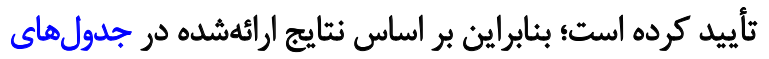

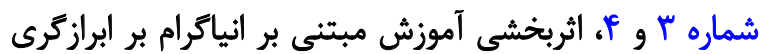
هيجانى مثقاضيان ازدواج مورد تأييد قرار مى ثيثيرد.

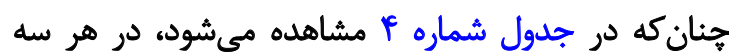

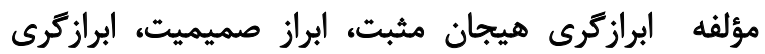

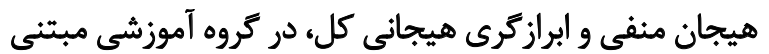

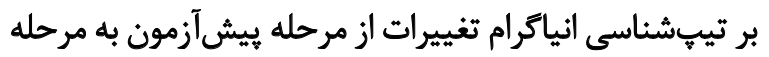

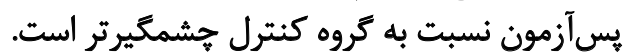

औ?

يروهش حاضر با هدف تأثير آموزش شخصيتمحور مبتنى

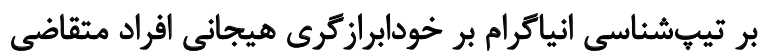

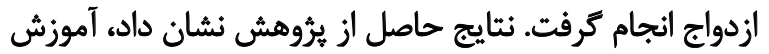

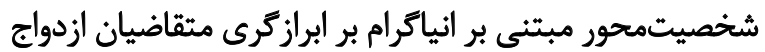

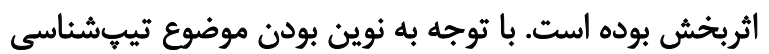

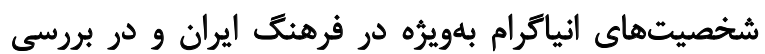

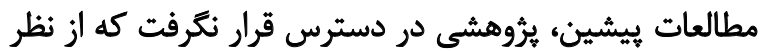

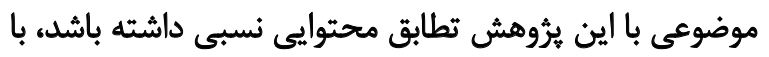

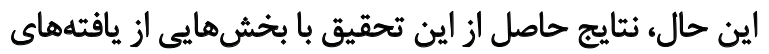

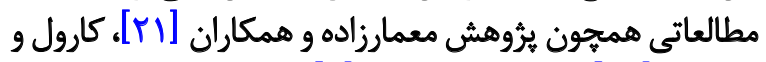

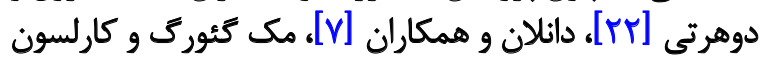

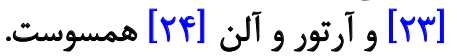

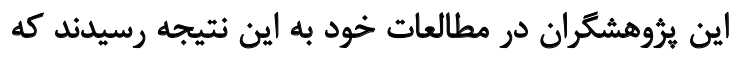

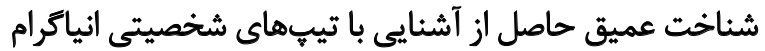

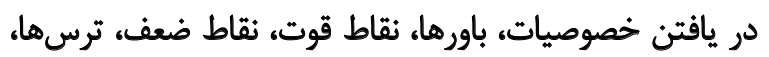

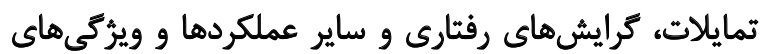

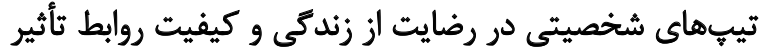

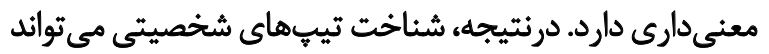

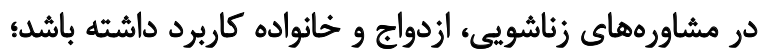

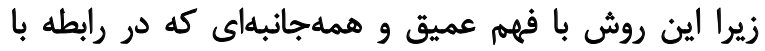

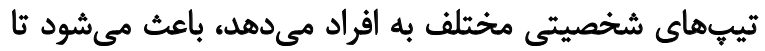

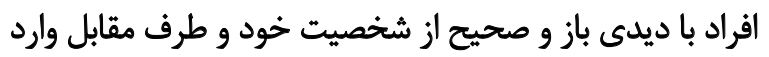

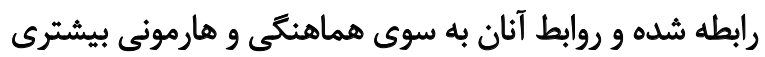

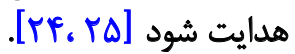

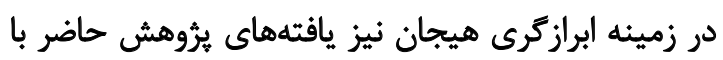

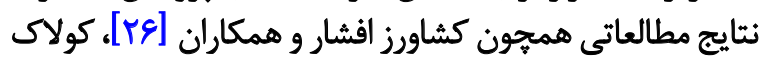

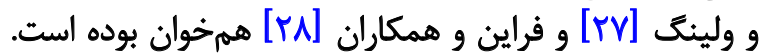

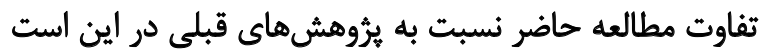

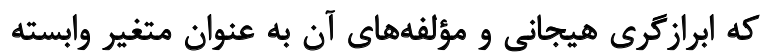

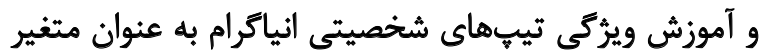

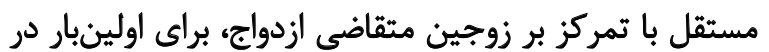
ايران به مرحله اجرا درآمده است زتئ. 
يك روش مناسب براى زوجين متقاضى ازدواج، كمـك بهآ آنها

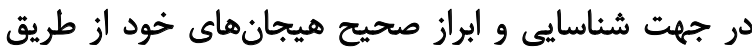

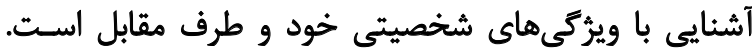

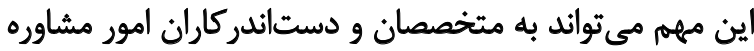
بيش از ازدواج جهت ارائه خدمات بهتر كمك كند.

ازي سوى ديكر بسته مشاوره قبل از ازدواج مبتنى بر تيبشناسى

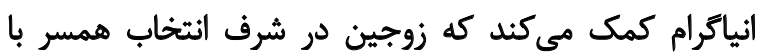

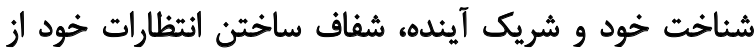

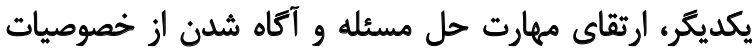

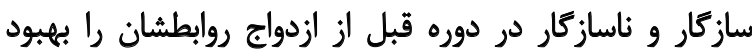

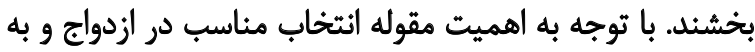

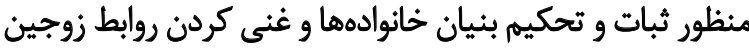

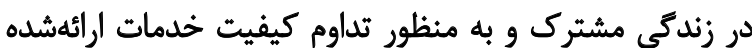

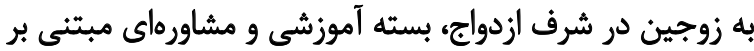

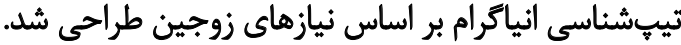
هر يثووهشى در مراحل مختلف خود با محدوديتهايى مواجه

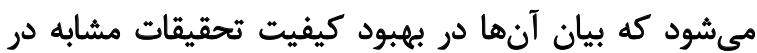

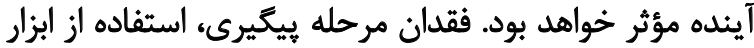

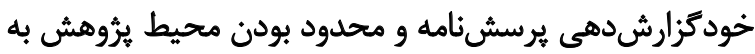

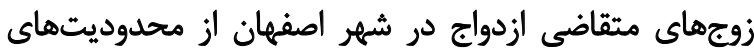

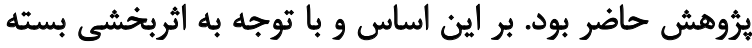

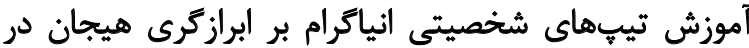

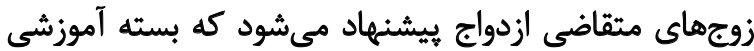

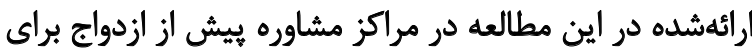

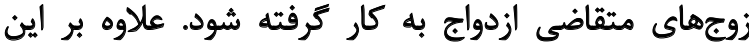

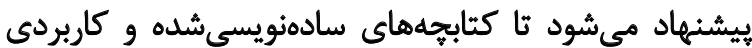

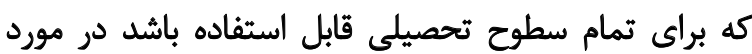

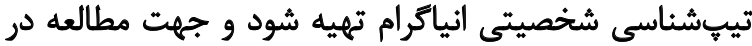
اختيار زوجين در مرحله بيش از ازدواج قرار تيردا

مالاحظات اخلاقي

\section{يبيروى ازاصول اخلاق يُوهش}

از به منظور رعايت مسائل اخلاقيى يس از آكاهى شركت كنيندكان

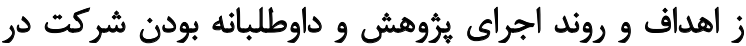

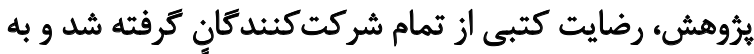

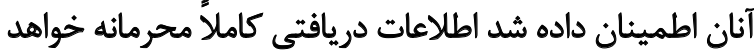

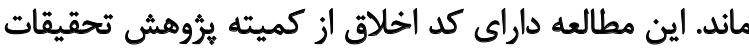
علمى (IR.IAU.KHUISF. REC. 1397.058) است.

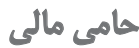

بنابر اظهار نويسنده مسئول، مقاله حامى مالى ندارد.
بر اساس برؤوهش اين محققان، از عوامل مؤثر درموفقيت

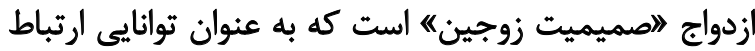
با ديكران با حفظ الفرديثه تعريف مىشيت الفود. اينكونه تعاريف

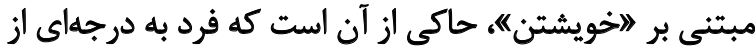

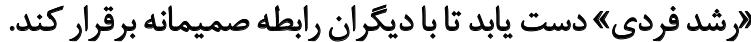

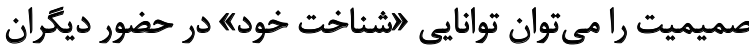

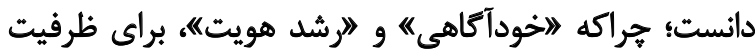

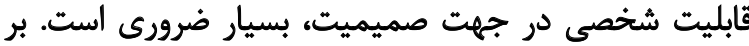

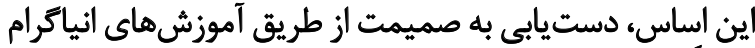

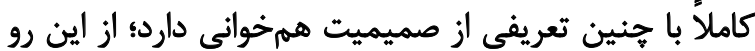

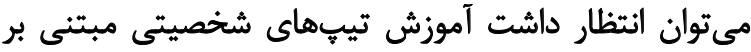

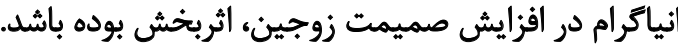

در تبيين اثربخشى آموزش تيبهاي شخصيتى بر ابرازكرى

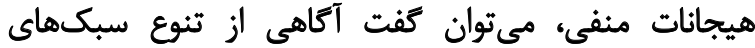

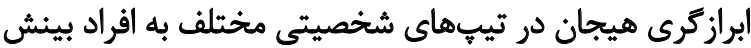

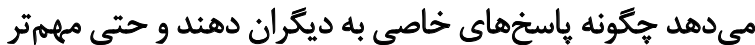

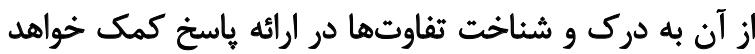

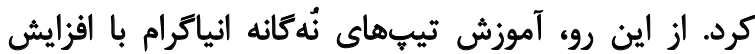

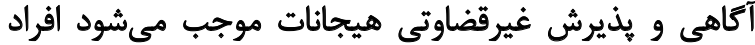

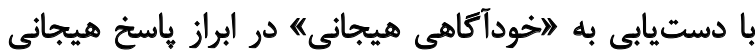

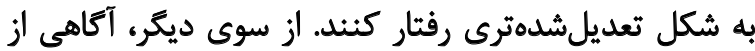

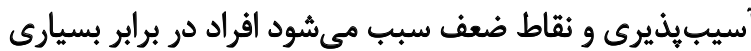

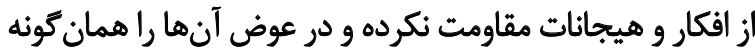

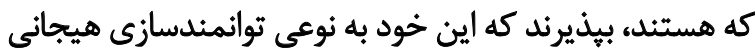

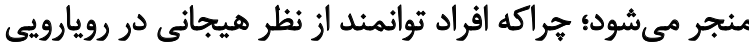

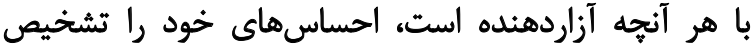

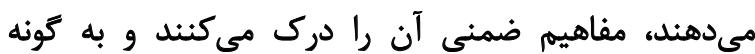

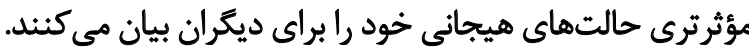
اين افراد در مقايسه با افرادى كه مهارت و توانايى ابراز مناسب

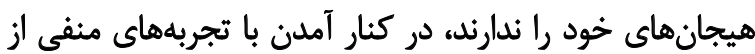

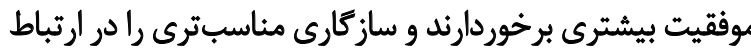

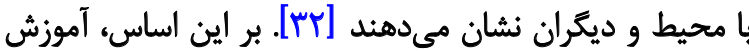

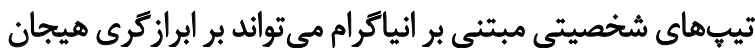

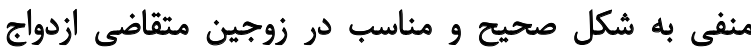

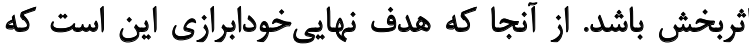

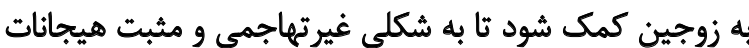

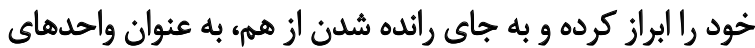

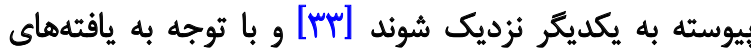

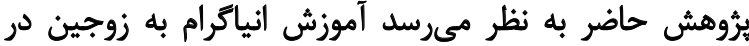

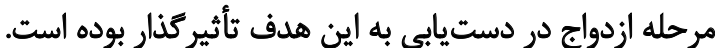
تيجيةكيرى

با در نظر كرفتن يافتههاى بُروهش حاضر، مىتوان كَفت 


$$
\text { هشاركت نويسندكان }
$$

مفهومسازى، تحليل داده، مديريت بروزه: تمام نويسندكان؛

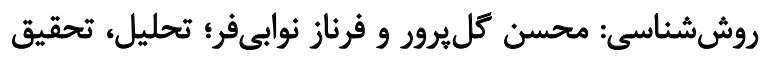

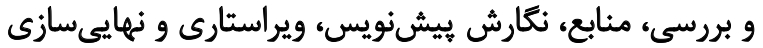
متن: فرئاز نوابى فئر.

$$
\text { تعارض مناقع }
$$

بنابر اظهار نويسندكان، اين مقاله تعارض منافع ندارد. 


\section{References}

[1] Kotrla K, Dyer P, Stelzer K. Marriage educatin with hispani couples: Evaluation of communication workshop. Family Science Review. 2010; 15(2):1-14. https:/ / www.semanticscholar.org/paper/Marriage-Education-with-Hispanic-Couples\%3A-of-a-Kotrla-Stelzer/a8a8196c34e85ea543dc9779c1c6b4a149901a61?p2df

[2] Doss BD, Rhoades GK, Stanley SM, Markman HJ, Johnson CA. Differential use of premarital education in first and second marriages. Journal of Family Psychology. 2009; 23(2):268-73. [DOI:10.1037/a0014356] [PMID]

[3] Risch GS, Riley LA, Lawer MG. Problematic issues in the early years of marriage: Content for premarital education. Journal of Psychology and Theology. 2003; 31(3):253-69. [DOI:10.1177/0091 64710303100310]

[4] Parhizgar O, Esmaelzadeh-Saeieh, S, Akbari Kamrani M, Rahimzadeh $\mathrm{M}$, Tehranizadeh $\mathrm{M}$. Effect of premarital counseling on marital satisfaction. Shiraz E-Medical Journal. 2017; 18(5):e13182. [DOI:10.5812/semj.44693]

[5] Naderi F, Eftekhar Z, Amolla Zadeh S. [The correlations among personality characteristics and intimate relationships with couple burnout in spouses of addict men in Ahwaz (Persian)]. Journal of Social Psychology (New Findings in Psychology). 2009; 4(11):6178. https:/ / www.sid.ir/Fa/Journal/ViewPaper.aspx?id=124229

[6] Sepehrian-Azar F, Galavandee H. [The effect of enneagram training on decrease of marital conflict (Persian)]. Journal of Family Counseling \& Psychotherapy. 2011; 1(2):161-70. https:/ / www. sid.ir/fa/journal/ViewPaper.aspx?ID=149096

[7] Brent Donnellan M, Conger RD, Bryant CM. Erratum to “The big five and enduring marriages" [Journal of Research in Personality 38 (2004) 481-504]. Journal of Research in Personality. 2005; 39(1):206-7. [DOI:10.1016/j.jp. 2004.10.001]

[8] Luo Sh, Klohnen EC. Assortative mating and marital quality in newlyweds: A couple-centered approach. Journal of Personality and Social Psychology. 2005; 88(2):304-26. [DOI:10.1037/00223514.88.2.304] [PMID]

[9] Claxton A, O'RourkeN,SmithJAZ, DeLongis A. Personality traits and marital satisfaction within enduring relationships: An intracouple discrepancy approach. Journal of Social and Personal Relationships. 2012; 29(3):375-96. [DOI:10.1177/0265407511431183]

[10] Abbasi R, Rasoulzadeh Tabatabaei SK. [Marital satisfaction and indivitual differences: The role of personality factors (Persian)]. International Journal of Behavioral Sciences. 2009; 3(3):237-42. https://www.sid.ir/fa/journal/ViewPaper.aspx?ID=99484

[11] Mohammadi A, Sahaghi H, Neysi M, Rezaee Nia Z. [Providing a preliminary model of the happily marriage: A qualitative study (Persian)]. Rooyesh-e- Ravanshenasi Journal. 2016; 6(3):135-62. http://frooyesh.ir/article-1-472-en.html

[12] Diefendorff JM, Richard EM, Yang J. Linking emotion regulation strategies to affective events and negative emotions at work. Journal of Vocational Behavior. 2008; 73(3):498-508. [DOI:10.1016/j.jvb.2008.09.006]

[13] Feldman Barrett L, Adolphs R, Marsella S, Martinez AM, Pollak SD. Emotional expressions reconsidered: Challenges to inferring emotion from human facial movements. Psychological Science in the Public Interest. 2019; 20(1):1-68. [DOI:10.1177/1529100619832930] [PMID] [PMCID]
[14] Konishi H, Froyen LG, Skibbe LE, Bowles RP. Family context and children's early literacy skills: The role of marriage quality and emotional expressiveness of mothers and fathers. Early Childhood Research Quarterly. 2018; 42:183-92. [DOI:10.1016/j. ecresq.2017.10.008]

[15] Pooralkhas Sh, Saljooghi Z. [Investigating the characters of the Miramar novel based on the Enneagram School (Persian)]. Paper presented at: $8^{\text {th }}$ Conference on Persian Language and Literature Research. January 2016; Tehran, Iran. https://www.sid.ir/Fa/ Seminar/ViewPaper.aspx?ID=31081

[16] Daniels DL, Price VA. The essential Enneagram: the definitive personality test and self-discovery guide [N. Seyed Mohammadi, Persian trans]. Seyed Mohammadi Y, editor. Tehran: Arasbaran; 2016. http://opac.nlai.ir/opac-prod/bibliographic/4616314

[17] Tallon R, Sikora M. Awareness to action: The Enneagram, emotional intelligence, and change. Scranton: University of Scranton Press; 2006. https://books.google.com/ books?id=BrC6AAAACAAJ\&dq

[18] Daghighian P. [Psychology of nine personalities: Enneagram (Persian)]. $2^{\text {nd }}$ ed. Tehran: Ashianeye Ketab; 2009. http://opac. nlai.ir/opac-prod/bibliographic/983829

[19] King LA, Emmons RA. Conflict over emotional expression Psychological and physical correlates. Journal of Personality and Social Psychology. 1990; 58(5):864-77. [DOI:10.1037/00223514.58.5.864]

[20] Rafiei Nia P, Rasoulzadeh Tabatabaei SK, Azad Fallah P. [Relationship between emotional expression styles and general health in college students (Persian)]. Journal of Psychology 2006; 10(1):84-105. https://www.sid.ir/fa/journal/ViewPaper. aspx?ID=49301

[21] Memarzadeh M, Khodabakhshi-Koolaee A, Khatiban M. [The effect of training the nine Enneagram personality types on spouse selection criteria and marital attitude of single girls (Persian)]. Journal of Education and Community Health. 2017; 3(4):24-30 [DOI:10.21859/jech.3.4.24]

[22] Carroll JS, Doherty WJ. Evaluating the effectiveness of premarital prevention programs: A meta-analytic review of outcome research. Family Relations. 2003; 52(2):105-18. [DOI:10.1111/j.17413729.2003.00105.x]

[23] McGeorge CR, Carlson TS. Premarital education: An Assessment of program efficacy. Contemporary Family Therapy. 2006; 28(1):165-90. [DOI:10.1007/s10591-006-9701-8]

[24] Arthur K, Allen K. The nature of love: Understanding the enneagram types as nine expressions of attachment. The Enneagram Journal. 2010; 3(1):6-22. http://iranenneagram.ir/wp-content/ uploads/2017/03/iranenneagram.c022.pdf

[25] Kashi G. [A survey of Enneagram training effectiveness on marital satisfaction of housewife self-esteeming in Tehran (Persian)] [MSc. thesis]. Tehran: Psychology of Al-Zahra University; 2010.

[26] Keshavarz-Afshar H, Abdini F, Ghahvehchi F, Asadi M, Jahanbakhshi $Z$. [The role of emotional expressiveness and demographic factors in predicting marital satisfaction among women (Persian)]. Semi-Annual Journal of Family Pathology, Counseling \& Enrichment. 2015; 1(1):59-66. http://fpcej.ir/article-1-106-fa. $\mathrm{html}$

[27] Kolak AM, Volling BL. Parental expressiveness as a moderator of coparenting and marital relationship quality. Family Rela- 
tions. 2007; 56(5):467-78. [DOI:10.1111/j.1741-3729.2007.00474.x] [PMID] [PMCID]

[28] Froyen LC, Skibbe LE, Bowles RP, Blow AJ, Gerde HK. Marital satisfaction, family emotional expressiveness, home learning environments, and children's emergent literacy. Journal of Marriage and Family. 2013; 75(1):42-55. [DOI:10.1111/j.17413737.2012.01035.x]

[29] O'Leary KD, Smith DA. Marital interactions. Annual Review of Psychology, 1991; 42:191-212. [DOI:10.1146/annurev. ps.42.020191.001203] [PMID]

[30] Moslemi M, Aghaei H. [The effectiveness of training based on group narrative therapy on the couples intimacy (Persian)]. Journal of Psychoscience. 2017; 16(62):265-82. http:/ / psychologicalscience.ir/article-1-152-en.html

[31] Patrick Sh, Sells JN, Giordano FG, Tollerud TR. Intimacy, differentiation, and personality variables as predictors of marital satisfaction. The Family Journal. 2007; 15(4):359-67. [DOI:10.1177/1066480707303754]

[32] de Veld DMJ, Marianne Riksen-Walraven J, de Weerth C. The relation between emotion regulation strategies and physiological stress responses in middle childhood. Psychoneuroendocrinology. 2012; 37(8):1309-19. [DOI:10.1016/j.psyneuen.2012.01.004] [PMID]

[33] Baron KG, Smith TW, Butner J, Nealey-Moore J, Hawkins MW, Uchino BN. Hostility, anger, and marital adjustment: Concurrent and prospective associations with psychological vulnerability. Journal of Behavioral Medicine. 2007; 30(1):1-10. [DOI:10.1007/ s10865-006-9086-z] [PMID] 